\title{
Preferences for the selection of unique tRNA primers revealed from analysis of HIV-I replication in peripheral blood mononuclear cells
}

\author{
Kenda L Moore-Rigdon¹, Barry R Kosloff², Richard L Kirkman² and \\ Casey D Morrow*2
}

Address: ${ }^{1}$ Department of Microbiology, University of Alabama at Birmingham, Birmingham, Alabama 35294, USA and ${ }^{2}$ Department of Cell Biology, University of Alabama at Birmingham, Birmingham, Alabama 35294, USA

Email: Kenda L Moore-Rigdon - kendalee@uab.edu; Barry R Kosloff - retrovir@uab.edu; Richard L Kirkman - kirkman@uab.edu;

Casey D Morrow* - caseym@uab.edu

* Corresponding author

Published: 24 March 2005

Retrovirology 2005, 2:21 doi:10.1186/1742-4690-2-21

This article is available from: http://www.retrovirology.com/content/2/I/2I

(C) 2005 Moore-Rigdon et al; licensee BioMed Central Ltd.

This is an Open Access article distributed under the terms of the Creative Commons Attribution License (http://creativecommons.org/licenses/by/2.0), which permits unrestricted use, distribution, and reproduction in any medium, provided the original work is properly cited.

\begin{abstract}
Background: All human immunodeficiency virus (HIV-I) uses a host tRNALys,3 as the primer for reverse transcription. The tRNALys,3 is bound to a region on the HIV-I genome, the primer-binding site (PBS), that is complementary to the 18 terminal nucleotides of tRNALys,3. How HIV-I selects the tRNA from the intracellular milieu is unresolved.

Results: HIV-I tRNA primer selection has been investigated using viruses in which the primerbinding site (PBS) and a sequence within U5 were altered so as to be complementary to tRNAMet, tRNAPro or tRNAlle. Analysis of the replication of these viruses in human peripheral blood mononuclear cells (PBMC) revealed preferences for the selection of certain tRNAs. HIV-I with the PBS altered to be complementary to tRNAMet, with and without the additional mutation in U5 to be complementary to the anticodon of tRNAMet, stably maintains the PBS complementary to tRNAMet following extended in vitro culture in PBMC. In contrast, viruses with either the PBS or PBS and U5 mutated to be complementary to tRNAlle were unstable during in vitro replication in PBMC and reverted to utilize tRNALys,3. Viruses with the PBS altered to be complementary to tRNA ${ }^{\text {Pro }}$ replicated in PBMC but reverted to use tRNA ${ }^{\text {Lys,3; }}$, viruses with mutations in both the U5 and PBS complementary to tRNAPro maintained this PBS, yet replicated poorly in PBMC.
\end{abstract}

Conclusion: The results of these studies demonstrate that HIV-I has preferences for selection of certain tRNAs for high-level replication in PBMC.

\section{Background}

Although the major steps in reverse transcription have been known for some time, the process by which the tRNA primer is specifically selected from the intracellular milieu by the virus is less well understood. Even though different retroviruses select different tRNA primers for reverse transcription, within a group of retroviruses, the tRNA primer selected is conserved [1,2]. For example, murine leukemia virus (MuLV) selects tRNAPro, while avian leukosis virus (ALV) selects tRNATrp $[3,4]$. Human immunodeficiency virus type 1 (HIV-1), as do all lentiviruses, selects $\mathrm{RRNA}^{\mathrm{Lys}, 3}$ for use as the primer for reverse 
transcription [5,6]. How and why HIV-1 exclusively selects tRNA ${ }^{\mathrm{Lys}, 3}$ as the primer for reverse transcription is not known. Pseudovirions composed of Gag and Gag-pol are enriched for tRNA ${ }^{\text {Lys }}$, including tRNA ${ }^{\text {Lys }, 3}$, that is used for initiation of reverse transcription $[2,7,8]$. Additional studies have shown that the specific incorporation of lysyl tRNA synthetase and its interaction with Gag could also be important for the specific capture of tRNA ${ }^{\text {Lys,3 }}$ used for initiation of reverse transcription [9-11].

Substitution of the primer-binding site (PBS) to be complementary to alternative tRNAs results in the capacity of HIV-1 to transiently use these tRNAs for initiation of reverse transcription [12-14], even though upon extended culture, these viruses all reverted back to specifically utilize tRNA ${ }^{\mathrm{Lys}, 3}$ for initiation of reverse transcription. In some instances, mutation of a region 5 ' of the PBS so as to be complementary to the anticodon of certain tRNAs, in conjunction with mutations of the PBS, results in the virus stably utilizing these alternative tRNAs for replication [1519]. Interestingly, analysis of the virion tRNAs of a virus that stably utilized tRNA ${ }^{\text {His }}$ for replication did not show a difference in composition compared to that of the wild type virus that utilized tRNA ${ }^{\mathrm{Lys}, 3}$, indicating that tRNAs in the HIV-1 virion did not necessarily reflect the tRNA selected for initiation of reverse transcription [20].

The fact that HIV-1 can select different tRNAs for replication suggests the virus has access to a variety of tRNAs. Recent studies from this laboratory demonstrated that the tRNA selected by HIV-1 for replication have undergone nuclear-to-cytoplasmic transport. Furthermore, the tRNAs that are aminoacylated, indicating inclusion in translation, are most efficiently selected as primers [21]. The realization that tRNA biogenesis and translation might intersect with primer selection has prompted us to reexamine the stability and replication of HIV-1 with a PBS complementary to alternative tRNAs in a relevant cell type peripheral blood mononuclear cells (PBMC). In a previous study, we found that HIV-1 in which the PBS was altered to be complementary to tRNA ${ }^{\mathrm{Lys} 1,2}$ or tRNA ${ }^{\text {His }}$ reverted to utilize tRNA ${ }^{\mathrm{Lys}, 3}$ upon extended culture in PBMC [22]. Viruses could be generated which stably utilized these tRNAs for replication if additional mutations within the U5, consisting of nucleotides complementary to the anticodon regions, were also included in the viral genomes. Interestingly, viruses which utilize tRNA ${ }^{\text {Lys } 1,2}$ had further adapted to utilize this tRNA, exhibiting replication characteristics similar to the wild type virus following extended in vitro replication in human PBMC. Similar results have been recently reported for HIV-1 in which the PBS and a second region upstream, the primer activation site (PAS), has been altered to be complementary to tRNA ${ }^{\text {Lys } 1,2}$ [23]. In this case, the virus stably utilized tRNA ${ }^{\mathrm{Lys} 1,2}$ for an extended culture period. A mutation in the RNase $\mathrm{H}$ domain of the reverse transcriptase was also found, although the major determinant of the stability of the PBS was correlated with the mutations in the PAS and PBS.

In the current study, we have further examined the preference of HIV-1 for certain tRNAs. A previous study from this laboratory has shown that viruses with a PBS complementary to tRNA ${ }^{\text {Pro }}$ or tRNA ${ }^{\text {Ile }}$ were unstable following replication in SupT1 cells, an immortalized, continuous, human $\mathrm{T}$ cell line [24]. However, during the process of reversion to a PBS complementary to tRNA ${ }^{\text {Lys }, 3}$, we noted several different anomalies with the PBS, including the isolation of viruses with multiple PBS complementary to other tRNAs and a virus in which the PBS was complementary to tRNA ${ }^{\text {Met. }}$. Further characterization of this virus revealed that it stably utilized tRNAMet as the initiation primer following additional mutations in which the U5 was made complementary to the anticodon region of tRNA $^{\text {Met }}[15,19]$. We have now analyzed the replication and stability of the PBS of viruses in which the PBS alone was altered to be complementary to tRNAMet, tRNA ${ }^{\text {Pro }}$ or tRNA ${ }^{\text {Ile }}$, as well as viruses with both the PBS and U5 region altered to be complementary to the 3' 18-nucleotides and anticodon of these tRNAs. Clear differences were identified with respect to primer preference that correlated with virus replication. The results of these studies therefore establish that preferences for selection of certain tRNAs to be used in reverse transcription by HIV-1 do exist and are more evident following replication in PBMC than in continuous $\mathrm{T}$ cell lines.

\section{Results}

Construction and characterization of HIV-I proviral genomes complementary to tRNAMet, $t R N A^{\text {Pro }}$ and tRNA $A^{\text {lle }}$ In previous studies, we have described the construction of HIV-1 proviral genomes in which the PBS was made complementary to alternative tRNAs [15-19]. For these studies, the proviral genomes were based on HXB2, which allows high-level replication in continuous $\mathrm{T}$ cell lines (eg. SupT1s). For the current studies, we have transferred the 5' LTR up to the BssHII site (nucleotide 233) from these clones into the NL4-3 proviral clone of HIV. The NL4-3 proviral clone of HIV-1, in contrast to the HXB clone, contains open reading frames for all the accessory proteins and replicates to high levels in PBMC. The U5-PBS regions of the subsequent proviral constructs, named pNL4-3Met, pNL4-3-Pro and pNL4-3-Ile were sequenced prior to analysis to confirm that the constructs were isogenic with the wild type with the exception of the 3' 18-nucleotide $\mathrm{PBS}$ region (Figure 1A).

To characterize these viruses, we first measured the production of infectious virus and p24 antigen following transfection into $293 \mathrm{~T}$ cells. Since $293 \mathrm{~T}$ cells do not 


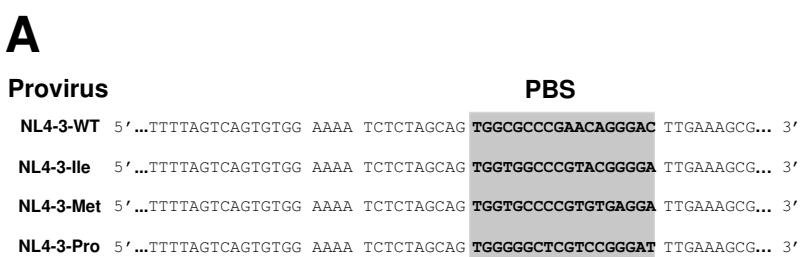

B Infectivity (\% Wild Type)

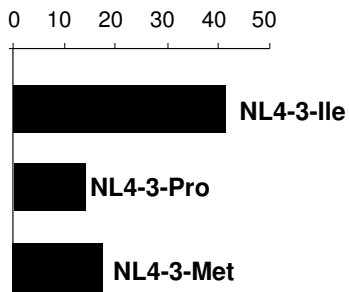

Figure I

U5-PBS sequence and infectivity levels of HIV-I NL43 viral mutants at start of PBMC infection. Panel $A$. HIV-I U5 and PBS sequence shown (from 5' to 3'). Viral primer binding site (PBS) sequence was altered to be complementary to the $3^{\prime}$ terminal 18 nucleotides of RRNA $^{\text {lle, }}$ tRNAMet and tRNAPro. The PBS sequence is shadowed.

Panel B. Comparison of infectivity of NL4-3 PBS mutants. HIV-I NL4-3 proviral clones were transfected into 293T cells, incubated for 48 hours, and supernatants were measured for infectious units. For a given sample, the number of infectious units per microliter is equal to the number of blue cells in a well divided by the dilution factor for that well and represents the average of at least two wells. Wild type infectivity levels were set at $100 \%$ and mutant virus infectivity was reported as a percentage of wild type. All viruses with altered PBS sequences showed reduced levels of infectivity as compared to wild type. Results presented are representative of three experiments.

support HIV-1 replication, this analysis would provide us with the inherent infectivities of viruses prior to undergoing reverse transcription/replication in PBMC. In our previous studies, we noted that there was no substantial difference in the production of virus (as measured by p24 antigen) as a result of altering the PBS in the HXB2 proviral constructs [15-19]. For the current studies, we transfected the proviral clones into $293 \mathrm{~T}$ cells and determined the amount of infectious units using the JC53BL assay; virus production was then measured using a p24 antigen capture ELISA. Infectivity was determined as the ratio of infectious units to p24 antigen. The values are presented relative to the infectivity of the wild type virus, with a PBS complementary to $\mathrm{RNA}^{\mathrm{Lys}, 3}$ (Figure $1 \mathrm{~B}$ ). All of the viruses with altered PBS had infectivities lower than the wild type. The virus NL4-3-Ile, with a PBS complementary to tRNAIle, was consistently the most infectious of the

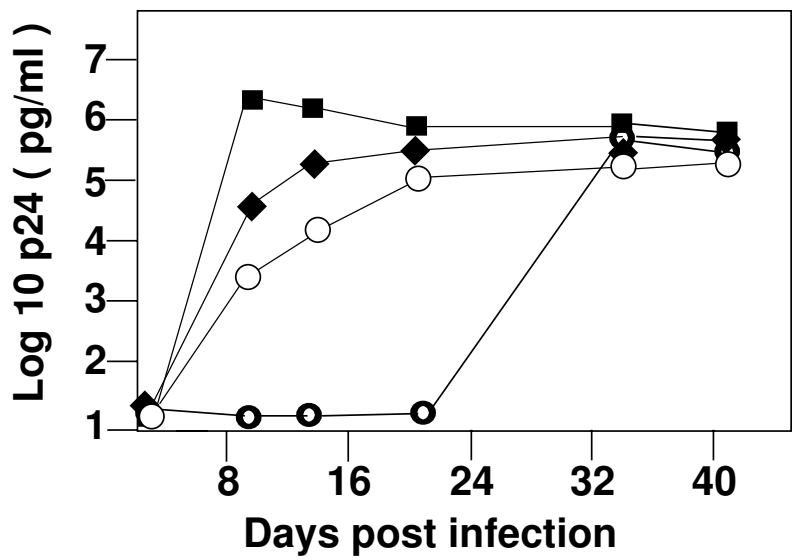

Figure 2

Replication of HIV-I with PBS sequence altered to be complementary to the 3' I 8 nucleotides of tRNAlle, tRNAMet and tRNAPro in human peripheral blood mononuclear cells (PBMC). Infections were initiated with transfection supernatant containing approximately $200 \mathrm{pg}$ of p24 antigen in a volume of $10 \mathrm{mLs}$ of media, giving a final p24 level of $20 \mathrm{pg} / \mathrm{mL}$ on day zero. At 14 day intervals, $5 \times 10^{6}$ fresh PHA stimulated PBMC were added to each culture. Supernatants were assayed for p24 viral antigen using an ELISA. Two additional separate infections produced similar replication patterns for each virus. Squares are wild type NL4-3; diamonds are NL4-3-Met; open circles are NL4-3-Ile and closed circles are NL4-3-Pro.

mutants with a level approximately $40 \%$ that of wild type, while the other viruses were $10-20 \%$ as infectious as the wild type virus.

\section{Stability of PBS following replication in PBMC}

We next wanted to determine the effects of alteration of the PBS on the replication of these viruses in PBMC. Infections were initiated with 200 pg of p24 and were allowed to proceed with re-feeding of PBMC every 14 days for periods of time exceeding 50 days of in vitro culture. The cultures were sampled periodically, supernatants were assayed for p24 antigen and cells were processed to extract high molecular weight DNA to determine the stability of the PBS. All of the viruses with an altered PBS showed an initial delay in production of p24 antigen compared to the wild type virus, consistent with the initial reduced infectivity compared to wild type (Figure 2). The NL4-3-Met virus had replication kinetics most similar to wild type virus in that during the first 10 days of culture we observed a rapid rise in p24 antigen, followed by a plateau at a level similar to that for wild type. The NL4-3-Ile virus replicated more slowly, with a gradual rise in p24 antigen before finally reaching a level similar to wild type. Finally, the 
Table I: Stability of PBS following extended culture in PBMC

\begin{tabular}{lll}
\hline Virus & PBS Sequence & Time to Reversion'(days) \\
\hline NL4-3-Ile & ${\text { Lys }, 3^{2}}^{2}$ & 21 \\
NL4-3-Met & Met $^{3}$ & --4 \\
>NL4-3-Pro & Lys, 3 & 35 \\
\hline
\end{tabular}

I. PBS analyzed at the time of in vitro culture in PBMC and found to be wild type, complementary to tRNALys,3.

2. PBS complementary to tRNALys,3.

3. PBS complementary to tRNAMet.

4. Analysis of 34 TA clones of the PBS following 63 days in culture revealed all maintained a PBS complementary to tRNA ${ }^{\text {Met. }}$

NL4-3-Pro virus showed minimal replication in the first 21 days of culture, followed by a rapid increase in the next 14 days to reach levels similar to that of the wild type virus (Figure 2). Although all of the viruses replicated in PBMC, the kinetics did not correlate with the initial infectivities from the 293T transfection supernatants.

During the culture period, we collected the DNA from the cells to determine the status of the integrated virus PBS. The wild type virus, as expected, maintained a PBS complementary to tRNA ${ }^{\mathrm{Lys}, 3}$ throughout the entire culture period (data not shown). In contrast, viruses with a PBS complementary to tRNA ${ }^{\text {Ile }}$ initially used tRNA ${ }^{\text {Ile }}$ for reverse transcription, but by day 21 from the initiation of culture had reverted to be complementary to tRNA ${ }^{\mathrm{Lys}, 3}$ (Table 1 ). Viruses in which the PBS was altered to be complementary to tRNAPro (NL4-3-Pro) appeared to be slightly more stable and maintained the PBS complementary to this tRNA through day 21 of culture, before reverting to wild type by day 35 . However, the subsequent rapid replication of the virus corresponded with the presence of a PBS complementary to tRNA ${ }^{\mathrm{Lys}, 3}$ (Table 1 ).

Surprisingly, the viruses in which the PBS was complementary to tRNA ${ }^{\text {Met }}$ remained stable for use of tRNA ${ }^{\text {Met }}$ throughout the complete culture period (in this case, up to 63 days post initiation of culture). Analysis of 34 individual TA clones of the PBS from these viruses revealed that all were complementary to tRNAMet (Table 1). This is the first instance in which we have found a virus that stably maintains a PBS complementary to an alternative tRNA (not tRNA ${ }^{\mathrm{Lys}, 3}$ ) following extensive in vitro replication that did not have additional mutations in U5. Previously, analysis of this virus in the HXB2 proviral clone revealed that the PBS was unstable following replication of the virus in vitro in SupT1 cells and reverted back to use tRNA $^{\text {Lys, } 3}[15,18]$. We further characterized the replication of this virus compared to wild type virus at different times during the culture period. Analysis of p24 antigen production from this virus at day 56 post initiation of culture revealed that it replicated similar to the wild type virus,
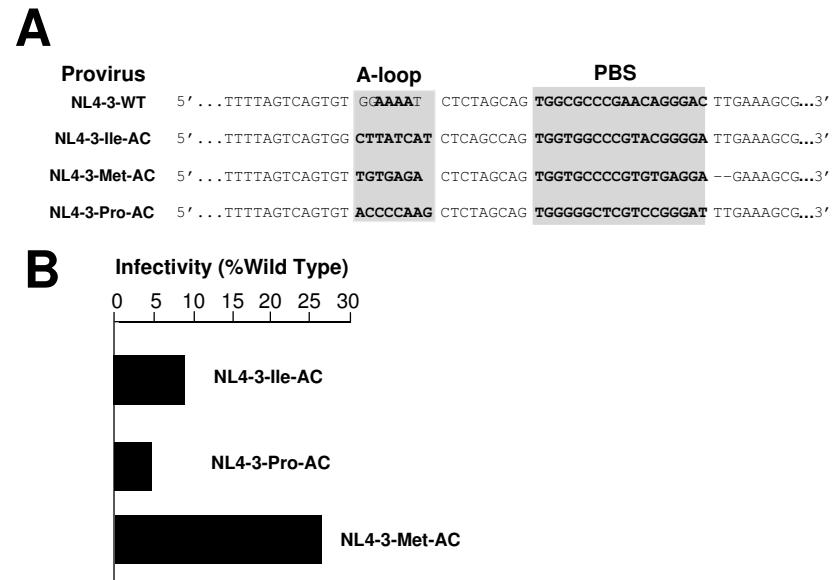

\section{Figure 3}

U5-PBS sequence and Infectivity levels of HIV-I NL43 viral mutants with altered $U 5$ and PBS sequences at start of PBMC infection. Panel A. HIV-I U5 and PBS sequence (shown $5^{\prime}$ to $\left.3^{\prime}\right)$. Viral primer binding site (PBS) and U5 A-loop sequences were altered to be complementary to the 3 ' terminal I 8 nucleotides and anticodon loop of tRNA ${ }^{\text {lle, }}$, tRNAMet, tRNAPro, and tRNATrp, respectively. U5 A-loop sequence and PBS are shadowed. Panel B. Comparison of the infectivity of U5-PBS mutant NL4-3 viruses. HIV-I NL4-3 proviral clones were transfected into 293T cells, incubated for 48 hours, and supernatants were measured for infectious units. For a given sample, the number of infectious units per microliter is equal to the number of blue cells in a well divided by the dilution factor for that well and represents the average of at least two wells. Wild type infectivity levels were set at 100\%, and mutant virus infectivity was reported as a percentage of wild type. All viruses with altered U5 and PBS sequences had reduced levels of infectivity as compared to wild type virus. The data presented are representative for three independent experiments.

albeit with slightly lower levels of p24 antigen (data not shown). The infectivity of the virus obtained after longterm culture, which utilized tRNA ${ }^{\mathrm{Met}}$, was approximately $50-80 \%$ of the wild type virus (data not shown). Collectively, the results of these studies establish that HIV-1 has a preference for certain tRNAs, such as tRNAMet, for the selection as primer for reverse transcription.

\section{Effect of mutations in U5 on replication of viruses that use alternative tRNAs}

In previous studies, we have found that with a PBS complementary to tRNA ${ }^{\text {Lys1,2, }}$ tRNA $^{\text {Met, }}$ tRNA ${ }^{\text {Glu }}$ and tRNA ${ }^{\text {His, }}$ the additional mutation in which the $\mathrm{U} 5$ region was made complementary to the anticodon stabilized the HXB2 proviral clones to allow continuous use of the alternative 


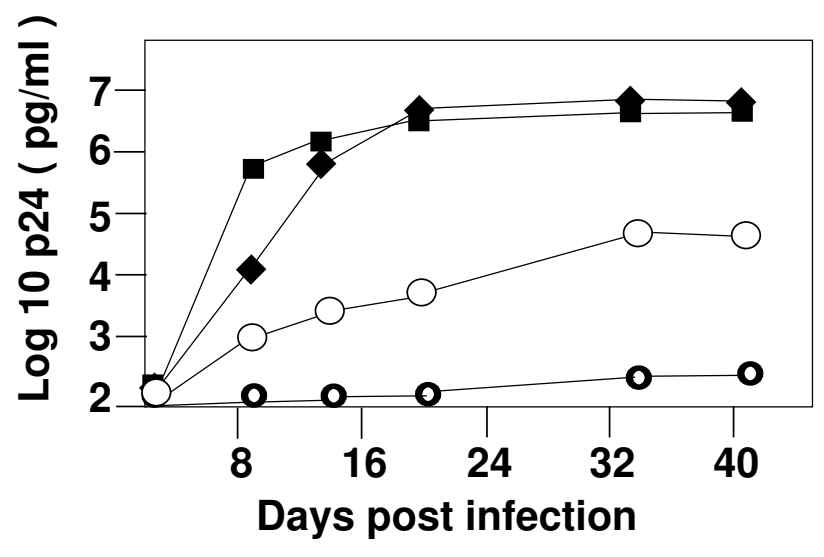

Figure 4

Replication of HIV-I with U5 and PBS sequence altered to be complementary to the anticodon loop and 3' I 8 nucleotides of tRNA ${ }^{\text {lle, tRNA }}$ (Ret, tRNAPro and tRNATrp in PBMC. Infections were initiated with transfection supernatant containing approximately $200 \mathrm{pg}$ of p24 antigen in a volume of $10 \mathrm{mLs}$ of media, giving a final p24 level of $20 \mathrm{pg} / \mathrm{mL}$ on day zero. Supernatants were assayed for p24 viral antigen every 7 days for a period of 42 days. At 14 day intervals, $5 \times 10^{6}$ fresh PHA stimulated PBMC were added to each culture. Two additional separate infections produced very similar replication patterns for each virus (data not shown). Squares are NL4-3 wild type; diamonds are NL4-3-Met-AC; open circles are NL4-3-Ile-AC; closed circles are NL4-3-Pro-AC.

tRNA during replication $[16,19,24,25]$. In contrast, in the HXB2 provirus, modification of the $\mathrm{U} 5$ region for viruses in which the PBS was made complementary to tRNAPro or tRNA $^{\text {Ile }}$ did not result in virus that could stably utilize these tRNAs following replication [24]. To determine if this would be case for viruses that were grown in PBMC, we constructed HIV-1 in which both the U5 and PBS were made complementary to tRNAMet, $\mathrm{tRNA}^{\text {Pro }}$ or $\mathrm{tRNA}^{\text {Ile }}$ (Figure $3 \mathrm{~A}$ ). The initial infectivities of the viruses were analyzed following transfection of the proviral clones into 293 cells. Similar to what we observed for viruses with just the PBS altered to be complementary to these tRNAs, the viruses with both the U5 and PBS altered demonstrated infectivities lower than wild type virus. In this case, the levels ranged from a low of 5\% (NL4-3-Pro-AC) to a high of $30 \%$ (NL4-3-Met-AC) (Figure 3B). We initiated infections in PBMC with the same amount of p24 antigen. We noted a delay in the production of p24 antigen in the cultures of viruses in which both the PBS and A loop were mutated to be complementary to the alternative tRNA ${ }^{\text {Met }}$ or tRNA ${ }^{\text {Ile, }}$, relative to the wild type virus (Figure 4). By day 21 , the viruses derived from pNL4-3-Met-AC had p24 antigen levels in the culture supernatants similar to that for the wild type virus. Viruses derived from pNL4-3-IleAC replicated at levels approximately $1 / 10$ that of the wild type virus, while viruses derived from pNL4-3-Pro-AC did not replicate well (or at all), as evidenced by p24 levels that did not increase substantially over the culture period (Figure 4).

We next analyzed the PBS of the viruses. Consistent with our previous studies, we found that the viruses in which both the U5 and PBS were complementary to tRNAMet remained stable during the culture period (Table 2). Sequence analysis of the virus that stably utilized tRNAMet (NL4-3-Met-AC) revealed a few nucleotide changes outside of the PBS. Previous studies from our laboratory have reported single nucleotide changes, noting that these changes might be important in stabilizing RNA structures to facilitate more effective primer selection [15,17-20,24]. Further experiments will be needed to address this issue. Characterization of NL4-3-Met-AC after extended culture revealed that it had infectivities that were still lower than that of the wild type virus (data not shown). In fact, the infectivities of the virus derived from pNL4-3-Met-AC were generally lower than those from the virus derived from pNL4-3-Met (data not shown). In contrast, viruses in which the PBS and U5 region were made complementary to tRNA ${ }^{\text {Ile }}$ were not stable and reverted to utilize tRNA ${ }^{\text {Lys, } 3}$ following in vitro replication (Table 2). Thus, the A loop modification did not stabilize the virus to continuously use tRNAlle for replication in PBMC. Virus with both the U5 and PBS altered to be complementary to tRNAPro replicated poorly in the in vitro culture. Amplification of the region containing the PBS required use of a double PCR method in which the initial PCR product was used as the template of the second reaction (double PCR). Sequence analysis revealed that NL4-3-Pro-AC had maintained a

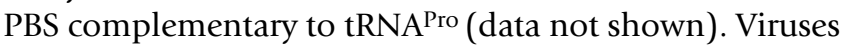
in which the U5 and PBS were made complementary to tRNA ${ }^{\text {Pro }}$ also reverted to use wild type following replication in SupT1 cells; in this case, we found viruses which contained multiple PBS, some of which were complementary to tRNA ${ }^{\mathrm{Lys}, 3}$. Following replication in PBMC, though, we did not isolate viruses with multiple PBS and all the viruses isolated contained a PBS complementary to tRN$\mathrm{A}^{\text {Pro }}$. Collectively, the results of these studies establish that HIV-1 does have a preference for tRNAMet over tRNAPro with respect to the selection of the tRNA primer for replication. Furthermore, tRNA ${ }^{\text {Ile }}$ is not favored for selection by HIV-1 even if compensatory mutations are provided in which the $\mathrm{U} 5$ region has been made complementary to the anticodon region.

\section{Discussion}

In previous studies, we have described HIV-1 in which the PBS and U5 have been altered to be complementary to 
Table 2: Analysis of U5-PBS from viruses following extended in vitro culture in PBMC

\begin{tabular}{|c|c|c|c|}
\hline Virus & U5-PBS & & $\begin{array}{l}\text { Days Post- } \\
\text { Culture }\end{array}$ \\
\hline & $11 e^{3}$ & & \\
\hline \multirow[t]{10}{*}{ NL4-3-Ile-AC } & '5' AGTCAGTGTTTATCAGCTCTAGCAG 2TGGTGGCCCGTACGGGGA TTGAAA 3' & Input 5 & 0 \\
\hline & lle & & \\
\hline & 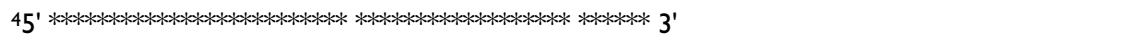 & PCR Product ${ }^{6}$ & 21 \\
\hline & Lys, 3 & & \\
\hline & 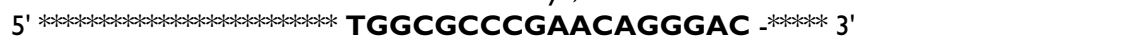 & 6/7 TA Clones ${ }^{7}$ & 35 \\
\hline & Lys, I,2 & & \\
\hline & 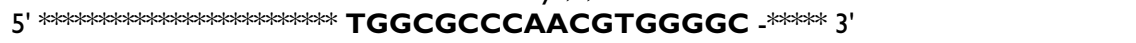 & I/7 TA Clones & 35 \\
\hline & Lys, 3 & & \\
\hline & $5^{\prime} * * * * * * * * * * * * * * * * * * * * * * * * *$ TGGCGCCCGAACAGGGAC $-* * * * * 31$ & PCR Product & 73 \\
\hline & Met & & \\
\hline \multirow{10}{*}{$\begin{array}{l}\text { NL4-3-Met- } \\
\underline{A C 5}\end{array}$} & 5' AGTCAGTGTTGTGAGACTGTAGCAG TGGTGCCCCGTGTGAGGC GAAAGC 3' & Input & 0 \\
\hline & Met & & \\
\hline & $5^{\prime} * * * * * * * * * * * * * * * * * * * * * * * * * * * * * * * * * * * * * * * * * * * * * * * * * * * * 31$ & $\begin{array}{l}\text { 5/10 TA } \\
\text { Clones }\end{array}$ & 35 \\
\hline & 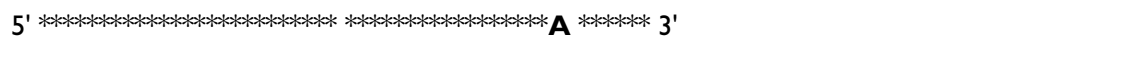 & $\begin{array}{l}\text { 3/10 TA } \\
\text { Clones }\end{array}$ & 35 \\
\hline & $5^{\prime} * * * * * * * * * * * * * * * * * * * * * * A^{*} * * * * * * * * * * * * * * * * * * * * * * * * * 31$ & $\begin{array}{l}\text { I/10 TA } \\
\text { Clones }\end{array}$ & 35 \\
\hline & 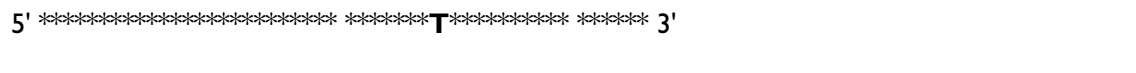 & $\begin{array}{l}\text { I/10 TA } \\
\text { Clones }\end{array}$ & 35 \\
\hline & Met & & \\
\hline & 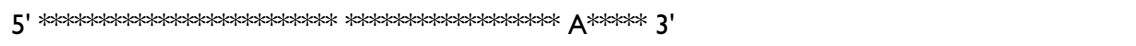 & 6/9 TA Clones & 63 \\
\hline & $5^{\prime} * * * * * * * * * * * * * * * * * * * * * * * * * * * * * * * * * * * * * * * * * * * * * * \mathbf{A} \mathrm{A}^{* * * * * *} 3^{\prime}$ & 2/9 TA Clones & 63 \\
\hline & 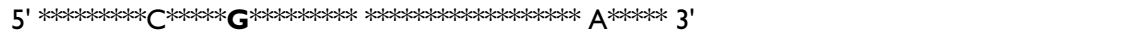 & I/9 TA Clones & 63 \\
\hline
\end{tabular}

I. The U5-loop is in bold type.

2. Spaces separate the PBS (indicated in bold type) from flanking sequence.

3. PBS complementary to the 3' terminal I8-nucleotide sequence of the indicated host tRNA.

4. Asterisks represent conserved nucleotides.

5. "Input" refers to the clone that was used to initiate viral infection in PBMCs.

6. PCR product that was sequenced directly.

7. Refers to TA clones of PCR product that is cloned into the Promega, P-Gem T-Easy Vector System I, to isolate individual colonies for sequencing.

tRNAMet, tRNA ${ }^{\text {Pro }}$ and tRNA ${ }^{\text {Ile }}[18,24]$. All of the viruses with only a PBS complementary to these tRNAs were replication competent but reverted to the wild type following infection in SupT1. To extend these studies to a more relevant cell type, we cloned the mutant PBS into the NL4-3 background, which replicates well in PBMC, reaching high levels of p24 antigen in the culture supernatant. Analysis of the effect of altering the PBS on infectivity of proviral clones revealed that these viruses were 10-40\% as infectious as the wild type virus, with the virus containing a PBS complementary to tRNA ${ }^{\text {Ile }}$ being the most infectious. However, analysis of the growth of these viruses revealed a clear preference for the viruses with a PBS complementary to tRNA ${ }^{\mathrm{Met}}$ compared to the virus with a

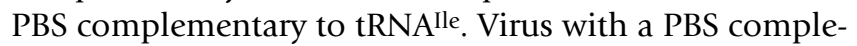

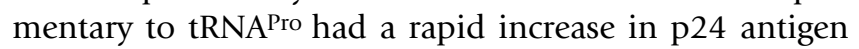
after 21 days in culture and subsequently replicated similar to wild type and viruses with a PBS complementary to tRNA ${ }^{\text {Met. }}$ As we had found in our previous studies, the sequence analysis of the PBS from both of these viruses at different times of culture revealed the reversion of the PBS to wild type [24]. The unexpected result from our studies was the distinct preference for HIV-1 to utilize tRNA ${ }^{\mathrm{Met}}$ as evidenced by the stability of the PBS following long-term culture. The preference of HIV-1 for the selection of tRNAMet was noted in a previous study in which we found a PBS complementary to this tRNA following analysis of the reversion of viruses that initially had a PBS complementary to tRNATrp [24]. A subsequent study found that HXB2 derived viruses in which only the PBS was mutated to be complementary to tRNAMet reverted back to the wild type PBS; a virus that could stably use tRNAMet was obtained by additional mutations in the U5 [15]. Thus, the results of our current study are unique in that the NL43-Met, without mutations in the U5, was stable and replicated well in PBMC, at a level comparable to the wild type virus. Further characterization of the viruses obtained from these two cell types will be needed to resolve the rea- 
son for differences in stability of the PBS. It is possible that differences in nucleotide concentrations or tRNA availability between the SupT1 or PBMC could influence the stability of the PBS. Further experiments using an endogenous RT reaction [18] and analysis of virus tRNA ${ }^{\text {Met }}$ content could be informative. With respect to the latter point though, our previous studies have not shown differences in tRNA content of virions that use alternative primers for reverse transcription [20].

How does this relate to the process of primer selection? In recent studies, we have found that HIV-1 most effectively selects tRNAs that have undergone the steps in tRNA biogenesis that result in transport from the nucleus to the cytoplasm [21]. Once in the cytoplasm, the tRNAs interact with a myriad of proteins involved in translation [26]. At any one time, the tRNA selected by HIV-1 as a primer for reverse transcription has been channeled into the translational process, supporting the idea of coupling of translation and primer selection. One possibility could be the coupling of primer selection with the synthesis of the Gagpol polyprotein. Previous studies have shown that pseudovirions composed of Gag and Gag-pol contain the appropriate ratios of tRNA ${ }^{\text {Lys }}$ found in intact wild type virions $[7,8]$. That is, during the translation of Gag-pol, the tRNAs available for selection might be enriched for tRNALys, 3 and tRNAMet; conversely, tRNA ${ }^{\text {Ile }}$ may not be favored because of the absence of isoleucine during translation of Gag-pol. This is not because isoleucine is excluded from the Gag-pol protein. Rather, it is possible that a translational event in the production of Gag-pol, possibly at or during the frame shifting, could influence the local amounts of tRNA so as to favor some (e.g., tRNA ${ }^{\mathrm{Lys}, 3}$, tRNA ${ }^{\mathrm{Met}}$ ) while not others (e.g., tRNA $\left.{ }^{\text {Ile }}\right)$. Without tRNA ${ }^{\text {Ile }}$ to occupy the PBS, there would be greater access by tRNALys, 3 to facilitate reversion back to the wild type PBS, complementary to tRNA $^{\mathrm{Lys}, 3}$. Viruses with a PBS complementary to RNAA $^{\text {Pro, }}$ and from previous studies those with PBS complementary to tRNA ${ }^{\text {His, }}$, tRNA ${ }^{\text {Lys } 1,2}$ or tRNAGlu, initially replicated slowly but reverted to use tRNA ${ }^{\text {Lys }, 3}$, whereupon they exhibited rapid replication. We would predict that the local availability of these tRNAs would be sufficient to allow the limited replication. However, given the selective pressure for the use of $\mathrm{tRNA}^{\mathrm{Lys}, 3}$, the virus would have a propensity to revert to wild type if the tRNAs were not present at levels similar to tRNA ${ }^{\text {Lys, }, 3}$ or tRNAMet. Coupling of the synthesis of Gag-pol with primer tRNA selection and encapsidation might provide all of the necessary components for the generation of infectious virus within the same intracellular locale. Further studies will be needed to explore the relationship between the synthesis of Gag-pol and primer selection using the unique viruses described in this study.
The results of our studies in which we included additional regions of complementarity between the tRNA and U5 further substantiates a role for this interaction in the selection of the tRNA primer [24]. In a recent study, we found that viruses with PBS and U5 complementary to tRNA ${ }^{\text {Lys } 1,2}$ or tRNA ${ }^{\text {His }}$ were stable after extended replication in PBMC, similar to what we found for NL4-3-Met-AC [22]. In this study, the virus derived from NL4-3-Pro-AC replicated poorly and in contrast to NL4-3-Pro, did not revert to wild type following extensive in vitro culture in PBMC. This finding supports the idea that the complementarity between the U5 and tRNA can impact the selection process. Most probably, the NL4-3-Pro-AC remains stable because it can more effectively select tRNA ${ }^{\text {Pro, }}$ or exclude tRNA ${ }^{\mathrm{Lys}, 3}$, from binding to the PBS complementary to tRNA $^{\text {Pro. If }}$ tRNA ${ }^{\mathrm{Lys}, 3}$ is used, the PBS generated during plus strand synthesis would be complementary to tRNA ${ }^{\mathrm{Lys}, 3}$, which could facilitate reversion upon subsequent replication. The results of the current study and others are consistent with the concept that multiple interactions between the viral RNA genome and tRNA occur during the selection process $[16,19,23,24]$. A recent study found that a virus that stably used tRNA ${ }^{\text {Lys } 1,2}$ could be generated by changing the PBS and a region upstream, different from the A loop, designated as the primer activation site (PAS) [23]. Interestingly, a virus with similar mutations to facilitate the use of tRNA ${ }^{\text {Pro }}$ was not stable, consistent with the results presented in our study. We suspect that the U5-PBS interactions are more important for tRNA selection in primary cells (e.g., PBMC) where the availability of the tRNAs in the intracellular environment might be different. Further experiments will be needed to address this issue.

In summary, the results of our studies analyzing the replication in PBMC of HIV-1 with PBS complementary to alternative tRNAs has revealed a clear preference for certain tRNAs to be selected for replication. The tRNA ${ }^{\text {Met }}$ is highly favored for selection, slightly less than the wild type tRNA ${ }^{\mathrm{Lys}, 3}$, while tRNA ${ }^{\text {Ile }}$ is not favored for selection as evidenced by the fact that viruses with this U5-PBS revert to use tRNA ${ }^{\text {Lys }, 3}$ after short term culture. Viruses that use tRNAs such as tRNA ${ }^{\text {Pro, }}$ tRNA ${ }^{\mathrm{Lys} 1,2}$ or tRNA ${ }^{\text {His }}$ replicate poorly in PBMC compared to the wild type virus and the virus that uses tRNA ${ }^{\text {Met }}$ [22]. These results suggest that HIV-1 can select the tRNA primer from a pool of tRNAs, with certain tRNAs favored over others, further substantiating a link between viral protein translation and primer selection.

\section{Conclusion}

The results of our study provide new insights into the tRNA selection process by HIV-1. For the first time, we have described a unique HIV-1 that utilizes a tRNA primer (tRNA ${ }^{\mathrm{Met}}$ ) that does not require additional mutations within the U5. This virus replicates well in human PBMC, 
similar to the wild type virus. In contrast, the virus did not prefer to select tRNA Ile as evidenced by the fact that this virus was unstable with or without additional mutations within U5. This result highlights that different tRNAs are available in PBMC for capture by HIV-1 for use as the primer for reverse transcription. The importance of additional mutations within $\mathrm{U} 5$ that are complementary to the anti-codon region of tRNAs for selection was highlighted by the studies with viruses in which the PBS was made

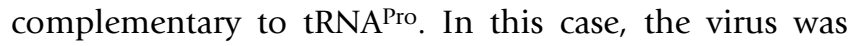
unstable with only the PBS complementary to tRNAPro while the additional $\mathrm{U} 5$ mutation did not allow reversion but severely impacted on the subsequent replication capacity of the virus, demonstrating that complex RNARNA interactions occur within the U5-PBS during primer selection. Collectively, the results of our studies demonstrate, for the first time, that distinct preferences exist for the selection of tRNAs to be used as the primer for HIV-1 reverse transcription. Coupled with our previous studies, we conclude there is most probably a link between viral translation and primer selection. The exclusive use of tRN$\mathrm{A}^{\mathrm{Lys}, 3}$ by HIV-1 is most probably due to inherent features of this tRNA as well as the intracellular availability during viral translation.

\section{Methods \\ Construction of NL4-3 proviruses containing modified PBS regions}

We previously reported the construction of pHXB2 (Met and Met-AC) and pHXB2 (Ile and Ile-AC) and PHXB2 (Pro and Pro-AC) with PBS and PBS-U5 changes complimentary to the respective tRNA $3^{\prime}$ and anticodon sequences $[15,18,19,24]$. These proviral mutants were constructed in the pHXB2 molecular clone of HIV-1. In this study, the NL4-3 molecular clone of HIV-1 was used as the proviral backbone DNA for the U5-PBS mutants [27]. Proviral clones pHXB2 (Met, Met-AC, Pro, Pro-AC, Ile and Ile-AC) from these previous studies were digested with HpaI and BssHII restriction enzymes (New England Biolabs, Beverly, MA) to release an 868-bp fragment that contained the 5' LTR, PBS, and leader region from the gag gene of HXB2. The HpaI site is located upstream of the 5' LTR within the flanking sequence, and the BssHII site is located downstream of the PBS within the viral genome, in the proximity of nucleotide 255 (5'GCGCGC-3'). Digests were run on a $1 \%$ Agarose gel (Amresco, Solon, $\mathrm{OH})$ to separate the $868 \mathrm{bp}$ U5-PBS fragment from the pHXB2 proviral DNA fragment. U5-PBS fragments were isolated using the Qiagen Gel Extraction kit (Qiagen, Valencia, CA) and cloned into the NL4-3 HIV-1 proviral plasmid using the same BssHII and HpaI restriction sites. All resulting NL4-3 constructs were verified by DNA sequencing to ensure the identity of the mutated sequence and the successful ligation of the U5-PBS fragment into the pNL4-3.

\section{Tissue Culture and DNA transfections}

Transfections were performed according to the protocol for the Fugene 6 Transfection Reagent (Roche Molecular Biochemicals, Indianapolis, IN). Briefly, $2 \mu \mathrm{g}$ of proviral plasmid DNA and $3 \mu \mathrm{L}$ Fugene reagent were added to 100 $\mu \mathrm{L}$ of Dulbecco's modified Eagle's Medium (no Fetal Bovine Serum) (Cellgro by Mediatech, Herndon, VA). This mixture was incubated at room temperature for approximately 45 minutes then added drop-wise to one well of a 6 -well plate containing $60 \%$ confluent 293 T cells in DMEM with $10 \%$ Fetal Bovine Serum (FBS). The transfections were incubated overnight at $37^{\circ} \mathrm{C}$ and the medium was replaced with fresh DMEM containing $10 \%$ FBS (Hyclone, Logan, UT). After 48-hours, all supernatants were collected and stored at $-80^{\circ} \mathrm{C}$. Supernatants from transfected cells were assayed for HIV-1 p24 antigen (Beckman Coulter, Miami, FL) and infectivity [28].

\section{PBMC Infections}

Human peripheral blood mononuclear cells (PBMC) were collected, stimulated using rIL-2 phytohemagglutinin (PHA) (Sigma, St. Louis, MO) and maintained as described previously [22]. Infections were performed by innoculating $20 \times 10^{6}$ PHA-stimulated PBMC with a volume of transfection supernatant containing $200 \mathrm{pg}$ of p24 antigen and incubating for 2 hours at $37^{\circ} \mathrm{C}$ and $5 \%$ $\mathrm{CO}_{2}$. Virus/PBMC mixtures were transferred to $25 \mathrm{~cm}^{2}$ tissue culture flasks, and the final volumes were adjusted to $10 \mathrm{~mL}$ with RPMI 1640, 1× (Cellgro by Mediatech, Herndon, VA) containing 15\% FBS (Hyclone, Logan, UT) and $30 \mathrm{U} / \mathrm{mL}$ rIL-2 (Sigma, St. Louis, MO).

Infected PBMC cultures were maintained for 10 weeks by replacing half the volume of medium every 7 days, without removing PBMC. Every 7 days, $1 \mathrm{~mL}$ of cell suspension was removed and centrifuged in an Eppendorf microcentrifuge at $24,000 \times g$ for 2 minutes. Supernatant was separated from the cell pellet and stored at $-80^{\circ} \mathrm{C}$ for further analysis by p24 ELISA and JC53BL infectivity assays. Cell pellets were also stored at $-80^{\circ} \mathrm{C}$ for isolation of high molecular weight DNA. Every 14 days an additional $5 \times 10^{6}$ PHA-stimulated PBMC were added to each culture.

\section{Infectivity Assay}

Levels of infectious virus (IU/ $\mu \mathrm{L})$ in both $293 \mathrm{~T}$ and PBMC culture supernatants were determined using the JC53BL assay as previously described $[22,28]$. For a given test sample, the number of infectious units per microliter is equal to the number of blue cells in a well divided by the dilution factor for that well and represents the average of at least two wells. 


\section{PCR analysis of integrated PBS-containing proviral DNA} Cell pellets from virus cultures were stored at $-80^{\circ} \mathrm{C}$, and isolation of high molecular weight genomic DNA was performed as described previously [22]. Approximately $2 \mu \mathrm{g}$ of each genomic DNA sample was PCR amplified using 5 pmole $/ \mu \mathrm{L}$ EcoRI (5'-CGGAATTCTCTCCTTCTAGCCTCCGCTAGTC-3') and 5 pmole/ $\mu \mathrm{L}$ SphI (5'-CCTTGAGCATGCGATCTACCACACACAAGGC-3') primers (Gibco BRL, Rockville, MD) with $2.5 \mathrm{mM}$ dNTP, $50 \mathrm{mM} \mathrm{MgC1}_{2}$ (Invitrogen, Grand Island, NY), and $5 \mathrm{U} / \mu \mathrm{L}$ Recombinant TAQ DNA polymerase (Invitrogen, Grand Island, NY). The PCR program used to amplify genomic DNA had a denaturation temperature of $94^{\circ} \mathrm{C}$ and an annealing temperature of $56^{\circ} \mathrm{C}$. The resulting PCR product was isolated and purified as described previously [22]. In cases of low virus replication (eg. pNL4-3-Pro-AC), the PCR product was used as a template for an additional PCR reaction (referred to as double PCR).

\section{Subcloning of PCR products and DNA Sequencing}

Purified PCR product was sequenced for the U5-PBS region of the viral genome using the EcoRI primer (Invitrogen, Grand Island, NY). DNA sequencing was performed on an automated DNA sequencer. PCR products that resulted in accordant sequence throughout the U5PBS region were considered to be a homogenous infection of virus, using the same tRNA primer. PCR products that resulted in discordant sequence in the PBS region were considered to be a heterogeneous population of virus, using more than one tRNA to prime reverse transcription, and was therefore, subjected to further TA cloning in order to isolate sequences of individual viruses. This PCR product was subcloned according to the Promega pGEM-T Easy Vector System I (Promega, Madison, WI) to prepare the DNA for efficient and accurate sequencing. PCR product was ligated into the pGEM-T Easy plasmid vector at $4^{\circ} \mathrm{C}$ overnight. Ligations were then transformed into $\mathrm{DH} 5 \alpha$ Escherichia coli cells (Invitrogen, Grand Island, NY) and grown overnight on LB with $100 \mu \mathrm{g} / \mathrm{mL}$ ampicillin and 20 $\mathrm{mg} / \mathrm{mL}$ Xgal. White colonies (indicating successful ligation) were picked and grown in LB-Amp100 $\mu \mathrm{g} / \mathrm{mL}$ broth overnight at $37^{\circ} \mathrm{C}$. DNA was harvested using the Qiagen QIAprep Spin miniprep kit, according to protocol (Qiagen, Valencia, CA). To assure that the TA clone DNA contained the PCR product insert, samples were digested by EcoRI to release the ligated fragment. Digests were run on a $1 \%$ agarose gel to verify the presence of a band of approximately 750 bp size. The TA clone DNA was then sequenced for the U5-PBS region using the EcoRI primer.

\section{Acknowledgements}

We would like to thank members of the Morrow laboratory for helpful discussion and Adrienne Ellis for preparation of the manuscript. KLM-R was supported by training grant (AI 07493). The UAB Center for AIDS Research Molecular Biology Core is acknowledged for help with the construction of the proviral clones (Al 27767). DNA Sequencing was carried out by Maria Salazar in the UAB CFAR DNA Sequencing Core (AI 27767). CDM acknowledges the helpful discussions from MAR. This research was supported by a grant from the NIH to CDM (Al 34749).

\section{References}

I. Marquet R, Isel C, Ehresmann C, Ehresmann B: tRNAs as primer of reverse transcriptases. Biochimie 1995, 77: I I3-I 24.

2. Mak J, Khorchid A, Cao Q, Huang Y, Lowy I, Parniak MA, Prasad VR, Wainberg MA, Kleiman L: Effects of mutations in Prl60gag-pol upon tRNALys,3 and Prl60gag-pol incorporation into HIV-I. I Mol Biol 1997, 265:4I 9-43I.

3. Harada F, Sawyer RC, Dahlberg JE: A primer ribonucleic acid for initiation of in vitro Rous sarcoma virus deoxyribonucleic acid synthesis. J Biol Chem 1975, 250:3487-3497.

4. Peters G, Harada F, Dahlberg JE, Panet A, Haseltine WA, Baltimore D: Low-molecular- weight RNAs of Moloney murine leukemia virus: Identification of the primer for RNA- directed DNA synthesis. J Virol 1977, 21:1031-104I.

5. Muesing MA, Smith DH, Cabradilla CD, Benton CV, Lasky LA, Capon DJ: Nucleic acid structure and expression of the human AIDS/lymphadenopathy retrovirus. Nature 1985, 3 1 3:450-458.

6. Ratner L, Haseltine W, Patarca R, Livak KJ, Starcich B, Josephs SF, Doran ER, Rafalski JA, Whitehorn EA, Baumeister K, et al.: Complete nucleotide sequence of the AIDS virus, HTLV-III. Nature 1985, 3 | 3:277-284.

7. Mak J, Jiang M, Wainberg MA, Hammarskjold M-L, Rekosh D, Kleiman $\mathrm{L}$ : Role of $\mathrm{Pr} / 60 \mathrm{gag}-\mathrm{pol}$ in mediating the selective incorporation of tRNALys into human immunodeficiency virus type I particles. J Virol 1994, 68:2065-2072.

8. Kohorchid A, Javannbakht $H$, Wise $S$, Halwani R, Parniak MA, Wainberg MA, Kleiman L: Sequences within Prl60gag-pol affecting the selective packaging of primer tRNALys,3 into HIV-I. J Mol Biol 2000, 299: 17-26.

9. Cen S, Javanbakht H, Kim S, Shiba K, Craven RC, Rein A, Ewalt KL, Schimmel P, Musier-Forsyth K, Kleiman L: Retrovirus-specific packaging of aminoacyl-tRNA synthetases with cognate primer tRNAs. J Virol 2002, 76: |3 || |-|3||

10. Jiang M, Mak J, Huang $Y$, Kleiman L: Reverse transcriptase is an important factor for the primer tRNA selection in HIV-I. Leukemia 1994, 8 SuppI I:SI49-SI5I.

II. Javanbakht H, Halwani R, Cen S, Saadatmand J, Musier-Forsyth K, Gottlinger H, Kleiman L: The interaction between HIV-I Gag and human lysyl-tRNA synthetase during viral assembly. J Biol Chem 2003, 278(30):27644-2765 I.

12. Wakefield JK, Wolf AG, Morrow CD: Human immunodeficiency virus type I can use different tRNAs as primers for reverse transcription but selectively maintains a primer binding site complementary to tRNALys,3. J Virol 1995, 69:6021-6029.

13. Li X, Mak J, Arts EJ, Gu Z, Kleiman L, Wainberg MA, Parniak MA: Effects of alterations of primer-binding site sequences on human immunodeficiency virus type I replication. J Virol I994, 68(10):6198-6206

14. Das AT, Klaver B, Berkhout B: Reduced replication of human immunodeficiency virus type I mutants that use reverse transcription primers other than the natural tRNA ${ }^{\text {Lys,3. }}$. J Virol 1995, 69(5):3090-3097.

15. Kang S-M, Zhang Z, Morrow CD: Identification of a sequence within $U 5$ required for human immunodeficiency virus type I to stably maintain a primer binding site complementary to tRNAMet. J Virol I997, 7 I(I):207-2I7.

16. Wakefield JK, Kang S-M, Morrow CD: Construction of a type I human immunodeficiency virus that maintains a primer binding site complementary to tRNAHis. J Virol 1996, 70:966-975

17. Zhang Z, Kang S-M, Li Y, Morrow CD: Genetic analysis of the U5PBS of a novel HIV-I reveals multiple interactions between the tRNA and RNA genome required for initiation of reverse transcription. RNA 1998, 4:394-406.

18. Kang S-M, Morrow CD: Genetic analysis of a unique human immunodeficiency virus type I (HIV-I) with a primer binding site complementary to tRNAMet supports a role for U5-PBS stem-loop RNA structures in initiation of HIV-I reverse transcription. J Virol 1999, 73:1818-1827.

19. Kang S-M, Zhang Z, Morrow CD: Identification of a human immunodeficiency virus type I that stably uses tRNALysI,2 
rather than tRNALys,3 for initiation of reverse transcription. Virology 1999, 257:95-105.

20. Zhang Z, Kang SM, LeBlanc A, Hajduk SL, Morrow CD: Nucleotide sequences within the $U 5$ region of the viral RNA genome are the major determinants for a human immunodeficiency virus type I to maintain a primer binding site complementary to tRNAHis. Virology 1996, 226:306-317.

21. Kelly NJ, Palmer MT, Morrow CD: Selection of retroviral reverse transcription primer is coordinated with tRNA biogenesis. J Virol 2003, 77:8695-870I.

22. Moore KL, Kosloff BR, Kelly NJ, Kirkman RL, Dupuy LC, McPherson S, Morrow CD: HIV type I that select tRNAHis or tRNALysI,2 as primers for reverse transcription exhibit different infectivities in peripheral blood mononuclear cells. AIDS Res Hum Retrov 2004, 20(4):373-38I.

23. Abbink TEM, Beerens N, Berkhout B: Forced selection of a human immunodeficiency virus type I that uses a non-self tRNA primer for reverse transcription: involvement of viral RNA sequences and the reverse transcriptase enzyme. J Virol 2004, 78(19): |0706-107|4.

24. Kang S-M, Wakefield JK, Morrow CD: Mutations in both the U5 region and the primer-binding site influence the selection of the tRNA used for the initiation of HIV-I reverse transcription. Virology 1996, 222:40I-4I4

25. Dupuy LC, Kelly NJ, Elgavish TE, Harvey SC, Morrow CD: Probing the importance of tRNA Anticodon: human immunodeficiency virus type I (HIV-I) RNA genome complementarity with an HIV-I that selects tRNA Glu for replication. J of Virol 2003, 77( I6):8756-8764

26. Arts G-J, Kuersten S, Romby P, Ehresmann B, Mattaj IW: The role of exportin-t in selective nuclear export of mature tRNAs. $E M B O$ J 1998, I7(24):7430-744I.

27. Adachi A, Gendelman HE, Koenig S, Folks T, Willey R, Rabson A, Martin MA: Production of acquired immunodeficiency syndromeassociated retrovirus in human and nonhuman cells transfected with an infectious molecular clone. J Virol 1986, 59(2):284-29I.

28. Derdeyn CA, Decker JM, Sfakianos JN, Wu X, O'Brien WA, Ratner L, Kappes JC, Shaw GM, Hunter E: Sensitivity of human immunodeficiency virus type I to the fusion inhibitor T-20 modulated by coreceptor specificity defined by the V3 loop of gp I 20. J Virol 2000, 74:8358-8367.
Publish with Bio Med Central and every scientist can read your work free of charge

"BioMed Central will be the most significant development for disseminating the results of biomedical research in our lifetime. "

Sir Paul Nurse, Cancer Research UK

Your research papers will be:

- available free of charge to the entire biomedical community

- peer reviewed and published immediately upon acceptance

- cited in PubMed and archived on PubMed Central

- yours - you keep the copyright 